\title{
Next Generation Transparent Supercapacitor Electrode Materials
}

\author{
Ziyan Fan
}

Irvine High School, Irvine, California, United States

\section{Introduction}

The transparent supercapacitor is a distinct type of supercapacitor that revolutionizes the electrochemical industry. It is developing toward computer glasses, wearing and portable electronics, or other highly advanced technological products. The transparency of electrode materia is directly proportional to its optical transmittance. Light waves with certain frequencies strike an object. If the object has the same frequency as the light, photons are absorbed and transformed into thermal energy of the vibrational movement. The light waves pass through the object with minimum reflection and re-emits on the other side of the object. This particular light wave is called a transmitted light wave. Reports have shown that an underlying transparent indium - tin oxide layer could provide efficient current collection and transparency. However, the element indium is expensive and unlikely to apply in the printed electronics in the future. Furthermore, its brittleness prevents applications where the flexibility of a supercapacitor is required. Graphene is a twodimensional layer with $\mathrm{sp}^{2}$ hybridized structure. It has strong mechanica strength, excellent flexibility, and good optical transparency. The implementation of chemical vapor deposition graphene film shows grea stretchability and transparency. And the inexpensive synthesis of graphene film of inexpensive $\mathrm{Ni}$ and $\mathrm{Cu}$ foils is a desirable approach. A wrinkled structure of graphene onto the polydimethylsiloxane (PDMS) showed less transparency and flexibility than that of the directly chemical-vapor-deposited layers of graphene film on the PDMS. Thus, the arrangement of graphene films also affects the transparency and flexibility of a supercapacitor. Carbon nanomaterials, generally have great flexibility and electric double-layer capacitance (EDLC) behaviors, showing wide applications in flexible supercapacitors. However, their optical transmittance is often limited by their low conductivity. It turns out its other replacement poly(3,4-ethylene dioxythiophene) polystyrene show more potential in the future mass production of transparen supercapacitors. PEDOT: PSS is a versatile electrode film. It not only fits the biosensor supercapacitor (in which it traps the virus and tests if the sample from a patient is contaminated by cancer cells or viruses), but it also suits, and commercially available for a transparent supercapacitor The PEDOT: PSS's volume alters the film transmittance, and the ransparency increases with the film transmittance. Experiments show that PEDOT: PSS is essentially better than most graphene electrodes, on the account that its superior performance attributes as a transparent supercapacitor charge storage film to its low optical conductivity. The PEDOT: PSS on the electrode is spin-coated from a doped PEDOT: PSS solution. Various asymmetric supercapacitors electrodes or hybrid electrochemical behavior. Nevertheless, the thick PEDOT: PSS film laye conveys lower optical transmittance that is contrary to a transparent supercapacitor The thangular Galvanic Chage-Discharge (GCD) shape of PEDOT. PSS indicates that it shows great capacilive behaviors as well. Polyvinylpyrrolidone (PVP) and $\mathrm{LiClO}_{4}$ electrolytes can also serve as the coating of a transparent supercapacitor. PVP is an amorphous polymer. It hood stability and displays moderate ionic conductivity. Whereas the of humidity and loss of residual solvents. These films have been employed in the fabrication of asymmetrical supercapacitor, which shows good transparency, capacitive behaviors, and some flexibility. The new devices illustrate the continued improvement in the development of transparent and flexible supercapacitors. In the future, researchers plan to further improve the energy density, flexibility, and durability, and stretchability of a supercapacitor.

\section{Results}

$\square$ Material

Material is the key indicator of the transparency, capacitance, and flexibility of a transparent supercapacitor electrode (TSE) material. Even though indium tin oxide (ITO) is still the most widely used TSE material nowadays, the material itself is brittle and expensive, which is not the most desirable material we want. Therefore, more materials emerge from studies of TSE around the globe. In general, the materials are categorized into two big groups. And these two groups extend many branches. The first big group is distinct materials. This section is divided into five large groups: ITO/FTO; 2. conductive polymers; 3 . Metallic nanostructures; 4 . Mxenes: 5 . carbon materials. The second big group is categorized by the different chemical configurations of a material. The most usual material configuration that is implemented onto the transparent supercapacitor (TSC) is the one-dimensional material (1D material), and two-dimensiona material (2D material). Three-dimensional materials (3D materials) are rarely used, but some of them convey high flexibility and optical transmittance due to their unique mechanical and optical properties. For technological advancement in the future, we seek better and more robus TSC material every day. ITO is widely used due to its high optical transmittance and electrical conductivity. It comprises of 74\% In, $18 \% \mathrm{O}$ and $8 \%$ Sn by weight. Its conductivity comes from the metallic bonds of indium doped tin oxide. On the other hand, its transmittance is high owing to its high light-scattering haze factor, which is the ratio of transmitted light and reflected light. The material looks like a piece of glass. Conductive polymers are another important material in the fabrication of TSE. They are characterized as robust, transparent, and highly conductive. Polymers are long organic chains. Picture a polymer as countless joints connected, since these are monomers with only a few nanometers. These joints give it multiple pivot points that enable it to move around quite easily. Therefore,
Young's modulus of the polymer materials is relatively high, which mean it has high flexibility. Polymers are transparent because the light can transmit through certain plastic materials. For example, polyethylene terephthalate (PET) is a common material to fabricate plastic bottles. Some polymers take thousands of years to decompose, which is environmentally unfriendly, so it's essential to choose the polymer materials we want wisely. The potential use of PEDOT: PSS conductive polymer films on a TSE intrigues many researchers. The PEDOT: PSS coated transparent

supercapacitor has electrodes that are $95 \%$ transparent, and a capacitance of $12 \mathrm{mF}$ at a scan rate of $50 \mathrm{mV} / \mathrm{s}$. Its capacitance is much higher than any previously reported transparent supercapacitor. This is where the third material nanostructures come in place. We create metallic nanowires on the surface of a substrate. The substrate is usually polymer to maximize its conductance. The thickness of the substance is inversely proportional to its reluctance. Thus, its thickness must be right to convey the maximum conductivity and transparency at the same time. The polymer can exist as either the current collector or the electrolyte to maximize the conductivity of a TSE. MXenes, which consist of few-atom-thick layers of transition metal carbides, nitrides, or carbonitride, are different from metallic nanostructures. Even though Mxenes contain metal compounds and are electrically conductive, it is, in fact, a 2D transition metal carbides and nitrides which have displayed promising properties in numerous applications, such as energy storage, electromagnetic interference shielding, and catalysis. A transparent, flexible, and conductive 2D titanium carbide (MXene) films display high volumetric capacitance . Its layered structure creates a good condition for intercalation reaction to take place. Therefore, it has a high energy density due to its high pseudocapacitance, and meanwhile a high power density. Last but not least, carbon materials are the second most abundant TSE materials due to their practicability They are characterized as highly flexible and conductive. Furthermore, 1D and $2 \mathrm{D}$ carbon materials show how

$\checkmark$ All in One Device

One way to further improve the TSE is to make a hybrid electrode materia that takes the advantages of different materials. The reason that we add metallic nanowires onto a thin polymer sheet is that we want the optimal electrical conductivity, optical transmittance, and flexibility altogether.

\section{Conclusions}

It's now time to take real consideration of the all in one device. Almost no materials are perfect alone, but if you choose the proper materials and combine them. Their advantages will compensate for their disadvantages. The hybrid supercapacitor electrode is the future of supercapacitor businesses. It will enable us to build better interactive devices such as smartphones, smart windows, smart glasses, tablets, and other touchable devices. In other words, the assembly of different materials maximizes the functionality of a transparent supercapacitor device. It merges the electrical conductivity, optical transmittance, and mechanical properties.

1. Compositing electrode materials make the best combination of high performance

Highly transparent and flexible electrolyte with safe and ionic

conductive performance is to be studied.

Metrics of quantifying transparency and flexibility of supercapacitors are to be developed.

In that way, future technologies like the ultrathin transparent tablets will show up soon because of the fabrication of an almost perfectly

transparent supercapacitor.

\section{Future Transparent Supercapacitors}

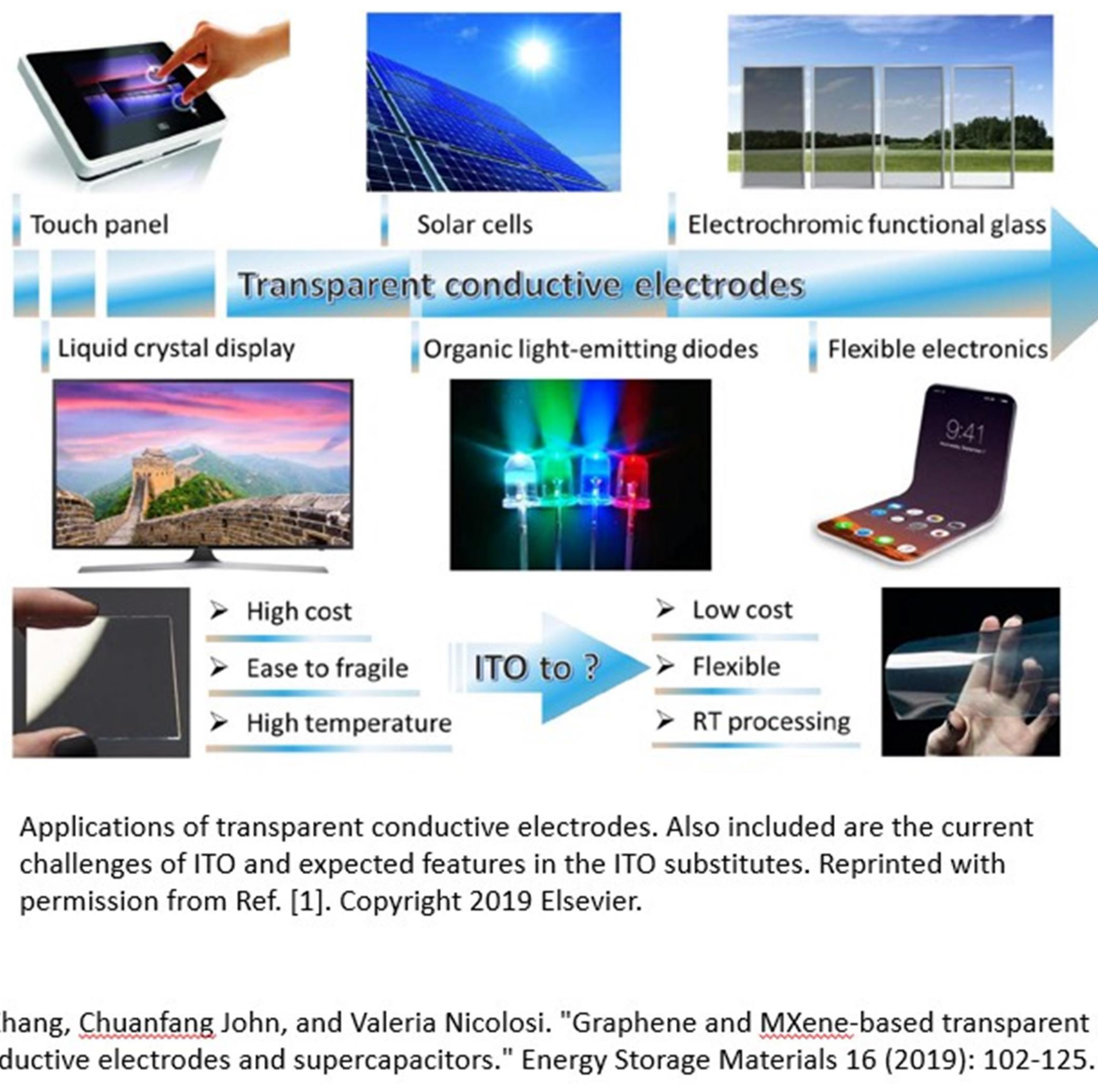

Bibliography

1. Ikhwan Ryu, MinHo Yang, Hyemin Kwon, Hoo Keun Park, Young Rag Do, Sang Bok Lee, and Sanggyu Yim, Coaxial $\mathrm{RuO}_{2}$-ITO Nanopillars for Transparent Supercapacitor Application, ACS, 2014.

2. Tao Chen, Yuhua Xue, Ajit K. Roy, and Liming Dai, Transparent and Stretchable High-Performance Supercapacitors Based on Wrinkled Graphene Electrodes, Acsnano, 2014

. Thomas M. Higgins and Jonathan N. Coleman, Avoiding Resistance Limitations in High-Performance Transparent Supercapacitor Electrodes Based on Large-Area, HighConductivity PEDOT: PSS Films, ACS Applied Materials, and Interfaces, 2015, 7. 